\title{
Prevalence of Pathogenic and Potentially Pathogenic Inborn Error of Immunity Associated Variants in Children with Severe Sepsis
}

Kate F. Kernan ( $\sim$ kate.kernan@chp.edu )

University of Pittsburgh https://orcid.org/0000-0002-6337-841X

Lina Ghaloul-Gonzalez

University of Pittsburgh, Pittsburgh

Jerry Vockley

University of Pittsburgh, Pittsburgh

Janette Lamb

University of Pittsburgh, Pittsburgh

Debborah Hollingshead

University of Pittsburgh, Pittsburgh

Uma Chandran

University of Pittsburgh, Pittsburgh

Rahil Sethi

University of Pittsburgh, Pittsburgh

Hyun-Jung Park

University of Pittsburgh, Pittsburgh

Robert A. Berg

Children's Hospital of Philadelphia, Philadelphia

David Wessel

Children's National Hospital, Washington

Murray M. Pollack

Children's National Hospital, Washington

Kathleen Meert

Central Michigan University

\section{Mark Hall}

Nationwide Children's Hospital Immune Surveillance Laboratory, and Nationwide Children's Hospital, Columbus

\section{Christopher Newth}

Children's Hospital Los Angeles, Los Angeles

John C Lin

St. Louis Children's Hospital, St. Louis 


\section{Allan Doctor}

St. Louis Children's Hospital, St. Louis

\section{Tom Shanley}

C. S. Mott Children's Hospital, Ann Arbor

\section{Tim Cornell}

C. S. Mott Children's Hospital, Ann Arbor

\section{Rick E. Harrison}

University of California Los Angeles

\section{Athena F. Zuppa}

Children's Hospital of Philadelphia, Philadelphia

\section{Russel Banks}

University of Utah

\section{Ron W. Reeder}

University of Utah

\section{Richard Holubkov}

University of Utah

Daniel A. Notterman

Princeton University, Princeton

\section{J. Michael Dean}

University of Utah

Joseph A. Carcillo

University of Pittsburgh

\section{Research Article}

Keywords: sepsis, inborn errors of immunity, hyperinflammation, primary immunodeficiency

Posted Date: November 2nd, 2021

DOI: https://doi.org/10.21203/rs.3.rs-366797/v2

License: (c) (1) This work is licensed under a Creative Commons Attribution 4.0 International License. Read Full License

Version of Record: A version of this preprint was published at Journal of Clinical Immunology on January 1st, 2022. See the published version at https://doi.org/10.1007/s10875-021-01183-4. 


\section{Abstract}

Purpose: Our understanding of inborn errors of immunity is increasing however, their contribution to pediatric sepsis is unknown.

Methods: We used whole exome sequencing to characterize variants in genes related to monogenic immunologic disorders in 330 children admitted to intensive care for severe sepsis. We defined candidate variants as rare variants classified as pathogenic or potentially pathogenic in Qiagen's Human Genetic Mutation Database or novel null variants in a disease-consistent inheritance pattern. We investigated variant correlation with infection and inflammatory phenotype.

Results: More than one in two children overall and three of four African American children had immunodeficiency-associated variants. Children with variants had increased odds of isolating a blood or urinary pathogen (blood: OR 2.82, 95\% Cl: $1.12-7.10, p=0.023$, urine: OR: 8.23, 95\% Cl: $1.06-64.11, p=$ 0.016 ) and demonstrating increased inflammation with hyperferritinemia (ferritin $\mathrm{ng} / \mathrm{mL}, \mathrm{OR}: 2.16,95 \%$

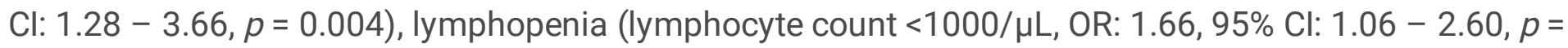
0.027), thrombocytopenia (platelet count $<150,000 / \mu \mathrm{L}, \mathrm{OR}: 1.76,95 \% \mathrm{Cl}: 1.12-2.76, p=0.013$ ) and CRP greater than 10mg/dL (OR: $1.71,95 \% \mathrm{Cl}: 1.10-2.68, p=0.017$ ). They also had increased odds of requiring extracorporeal membrane oxygenation (ECMO, OR: 4.19, 95\% Cl: $1.21-14.5, p=0.019$ ).

Conclusion: Herein, we describe the genetic findings in this severe pediatric sepsis cohort and their microbiologic and immunologic significance, providing evidence for phenotypic effect of these variants and rationale for screening children with life-threatening infection for potential inborn errors of immunity.

\section{Introduction}

Severe sepsis remains a leading cause of morbidity and mortality worldwide with 40 million annual cases, contributing to $60 \%$ of pediatric deaths, emphasizing a need for pathobiologic insight [1]. Inborn errors of immunity (IEI) are hypothesized to underlie vulnerability to life-threatening infection, not just in primary immunodeficiencies, but also in sporadic cases of severe sepsis [2]. While links have been explored in individual cases and pathogens such as influenza [3], invasive pneumococcus [4], Pseudomonas [5], SARS-CoV-2 [6,7], and previously healthy children with bacteremia [8], systematic investigation of IEI in pediatric sepsis is limited.

Next generation sequencing (NGS) advances have expanded our understanding of the molecular basis of IEI. Currently, the International Union of Immunologic Societies (IUIS) updates their catalogue of monogenic immunologic disorders biannually and describes over 400 genetic defects [9]. Due to phenotypic and genetic heterogeneity, whole exome sequencing (WES) is commonly used in their diagnosis. However, the broader application of WES to life-threatening infection has been hindered by challenges in variant interpretation. Additionally, even pathogenic variants are impacted by penetrance and expressivity and may not cause disease in all individuals [10]. This leads to a critical knowledge gap in understanding the prevalence and impact of IEI in severe pediatric sepsis. 
In the neonates, genetic disease contributes significantly to morbidity and mortality and NGS has demonstrated measurable clinical impact $[10,11]$. In older children with concern for genetic disease, diagnostic rates approach $40 \%$ and can be achieved in less than two weeks in research settings. The two largest NGS series in pediatric intensive care unit (PICU) patients both included cases of immunodeficiency with life-threatening infection $[12,13]$.

Building on these studies we used WES to test the hypothesis that variants in genes in the 2019 IUIS Primary Immunodeficiency classification are common among children with severe sepsis and associate with infectious and inflammatory phenotype.

\section{Methods}

\section{Subject Selection}

Children with severe sepsis between 44 weeks and 18 years old admitted to one of nine PICUs in the Eunice Kennedy Shriver National Institute of Child Health and Human Development Collaborative Pediatric Critical Care Research Network (NICHD-CPCCRN) between 2015-2017 without advance directives limiting care were eligible. The study was approved by the central and all nine site Institutional Review Boards. Written informed consent was obtained from one or more parents/guardians and child assent was garnered when able. At each center, all PICU admissions were screened twice weekly for the presence of sepsis, 1 organ failure and a central venous or arterial catheter. Study enrollment occurred a median of $2 \mathrm{~d}$ after ICU admission (IQR: +1 to $+4 \mathrm{~d}$ after ICU admission). Per site enrollment was limited to 80 subjects. Sepsis was defined as suspected or documented infection AND two Systemic Inflammatory Response criteria: 1 . Tachycardia (heart rate $>90$ th percentile for age); 2 . Tachypnea (respiratory rate $>90$ th percentile for age); 3 . Temperature $\left(<36^{\circ} \mathrm{C}\right.$ or $\left.>38.5^{\circ} \mathrm{C}\right)$; and, 4 . Abnormal WBC count $(>12,000 / \mu \mathrm{L}$ or $<4,000 / \mu \mathrm{L}$ or $>10 \%$ immature neutrophils). Severe sepsis was defined by the previously mentioned criteria AND at least one organ failure (cardiovascular: need for cardiovascular infusion support; pulmonary: need for mechanical ventilation support with $\mathrm{PaO} 2 / \mathrm{FIO} 2$ ratio $<300$ without support; hepatic: total bilirubin $>1.0 \mathrm{mg} / \mathrm{dL}$ and alanine aminotransferase $>100 \mathrm{IU} / \mathrm{L}$; renal: serum creatinine $>1.0 \mathrm{mg} / \mathrm{dL}$ and oliguria (urine output $<0.5 \mathrm{~mL} / \mathrm{kg} / \mathrm{hr}$ ); hematologic: thrombocytopenia $<100,000 / \mathrm{uL}$ and international normalized ratio >1.5x normal; and CNS: Glasgow Coma Scale <12 without sedatives) [14]. No geneticists or intensivists were involved in participant selection and suspicion for immunologic disorder was not considered at enrollment. Sequencing was performed between 2018-2020 and not available to treating physicians.

\section{DNA Extraction and Exome Sequencing}

A total of 401 pediatric patients were enrolled, for whom 381 parents (95\%) provided WES consent. $2 \mathrm{~mL}$ of whole blood were collected for DNA extraction using standard methods. 330 children completed WES (median DNA yield 39.24, IQR: 20.19 - 71.49 ) and 51 had insufficient DNA (median yield 1.120 , IQR: $0.205-3.455, p<0.0001$; Fig. 1). Those with insufficient DNA extraction had a lower median lymphocyte 
count on the day of sequencing blood draw ( 230 versus 1200 cells $/ \mathrm{ml}, p=1.43 \times 10^{-10}$ ), were older (median $8.5 y \vee 5.3 y, p=0.0008)$, less likely to be previously healthy $\left(13.7 \%\right.$ versus $\left.47.9 \%, p=2.8 \times 10^{-6}\right)$, more likely to have malignancy $\left(47.1 \%\right.$ versus $\left.7.0 \%, p=9.21 \times 10^{-12}\right)$, and suffered higher mortality $(23.5 \%$ versus $8.5 \%, p=0.0028)$.

The University of Pittsburgh Genomics Research Core performed WES on the lon Torrent platform. Libraries were constructed using the Ampliseq Exome RDY (ThermoFischer) with 100x target coverage. FASTQ files were aligned to Homo sapiens reference sequence GRCh37/hg19 to generate VCF files. Fabric Genomics' Opal software (Fabric Genomics Inc, CA) was used to identify nonsynonymous variants including missense, nonsense, frameshift, start or splice site (+/-2bp) mutations. Candidate variants were filtered for a minimum coverage $>10 x$ and a PHRED score $>20$ for quality control. No parental DNA samples were collected. Sanger sequencing proved prohibitive due to variant prevalence and was therefore not performed.

\section{Candidate Gene Filter}

The IUIS IEI report currently lists 430 genes as causes of 403 monogenic primary immunodeficiencies [9] categorized in nine groups: complement disorders, autoinflammatory disorders, combined immunodeficiencies with associated or syndromic defects, congenital defects of phagocyte number and function, disorders of immune dysregulation, defects in innate and intrinsic immunity, marrow failure, predominantly antibody deficiencies and immunodeficiencies affecting cellular and humoral immunity (Table S1). Candidate variants were restricted to this list, with minor allele frequency (MAF) $<0.05$ in ExAC, 1000 Genome, NHLBI-ESP 6500 and gnomAD databases. Variants were required to exhibit a disease-consistent inheritance pattern (one variant for autosomal dominant or X-linked in males, and two variants for autosomal recessive). We required only a single heterozygous variant for disorders with evidence supporting both recessive and dominant inheritance. Additionally, we limited missense variants to those classified as disease mutation (DM) or disease mutation? (DM?) in Qiagen's Professional Human Genetic Mutation Database (HGMD) based on peer-reviewed literature of the variant in human disease. The DM? designation indicates a potential but not definitive association and is an uncertain link between variant and disease. In this manuscript, DM and DM? variants are defined as pathogenic and potentially pathogenic respectively. As our dataset is without functional validation, we utilized HGMD for annotation where evidence for variant classification can be reviewed. We also aimed to increase power to detect genotype-phenotype associations in line with this resource's aim to minimize false negatives [16]. While HGMD professional is commonly cited as evidence for variant pathogenicity, it is not equivalent to clinical sequencing which integrates additional annotation resources including ClinVar and others. Therefore, while the amino acid changes in this manuscript are reported in peer-reviewed literature, they are not equated to immunodeficiency diagnosis. Complete references for individual variants are listed in table S2. Unique null (nonsense, frameshift, $+/-1$ or 2 splice site and initiation codon) variants found in a disease-causing inheritance pattern were treated as pathogenic per the American College of Medical Genetics (ACMG) standards and guidelines for variant interpretation [17]. Highly recurrent null 
variants were filtered from the dataset, due to high likelihood that they represented sequencing error or had no impact on function.

\section{Association Testing with Microbiologic and Inflammatory Markers}

We tested for association between any IEI variant or IUIS group and infection site and inflammatory markers. All microbiologic/virologic data were limited to the $48 \mathrm{~h}$ before or after enrollment. All results were reviewed, and likely contaminants were omitted by site including coagulase-negative staphylococci and viridans group streptococci blood cultures, Candida albicans and viridans group streptococci respiratory cultures, and mixed flora urine cultures. Culture-negative sepsis was defined as the absence of positive viral or bacterial testing from any site during the $48 \mathrm{~h}$ before or after enrollment after contaminant exclusion. For markers of inflammation, the most abnormal value per subject was used to define lymphopenia (minimum lymphocyte count $<1000 / \mu \mathrm{L}$ ), hyperferritinemia (ferritin $>500 \mathrm{ng} / \mathrm{mL}$ ), both markers of inflammation and risk for sepsis mortality [18,19], thrombocytopenia [20] (platelet count < $150,000 / \mu \mathrm{L}$ ) and extreme elevations in C-reactive protein (CRP > 10mg/dL, normal 0.04-0.79mg/dL) [21].

\section{Statistical Methods}

Comparisons between baseline characteristics and outcome were made between children with and without IEl using ${ }^{2}$ testing for categorical variables and Wilcoxon rank sum for continuous variables. For IUIS group comparisons to children without identified variants, Fisher exact testing was performed. For IUIS group comparisons, both unadjusted and adjusted $p$ values following multiple-test correction for the number of groups are reported (Benjamini-Hochberg method).

For individual identified variants, a cohort minor allele frequency (MAF) was computed and compared

using ${ }^{2}$ testing to gnomAD (https://gnomad.broadinstitute.org/v2.1.1) a sequencing database of 141,456 individuals that excludes those with severe pediatric diseases [22]. This approach has been used to identify overrepresented variants in rare disease cohorts [23]. Additional MAF comparisons were made based on self-reported race of any ethnicity between 1.) African American and non-African American children in the sepsis cohort, and 2.) African American children in the sepsis cohort and African participants in gnomAD. $P$ values were multiple-test corrected using the Benjamini-Hochberg method for the number of identified variants.

All statistics were completed in $\mathrm{R}$ version 4.0.4 using a $p$ value threshold of $<0.05$.

\section{Results}

\section{Prevalence of Inborn Error of Immunity Associated Pathogenic and Potentially Pathogenic Variants in Children with Severe Sepsis}

Among the 330 sequenced, variants occurred in 200 children (61\%) including 89 previously healthy children (Table 1). More than 3/4 of African American children with severe sepsis were found to have a 
genetic variant previously associated with IEI $(p=0.004)$. While most individuals harbored single IEI ( $N=104,52 \%$ of positive WES), 57 children (29\%) had two variants, 23 had three (12\%), and 16 had five or more (8\%, Fig. 2, Table S3). Additionally, children with IEI had increased odds of requiring extracorporeal membrane oxygenation (ECMO, OR 4.19,95\% Cl: 1.21 - 14.50, $p=0.019$ ). Other interventions including mechanical ventilation, continuous renal replacement therapy and plasma exchange did not differ between groups.

The variants exhibited both locus and allele diversity including 333 total variants at 131 loci (Fig 3.). Among these loci, 69 were associated with autosomal dominant disorders ( $N=144$ individuals), 25 with autosomal recessive conditions (homozygous or compound heterozygous in $\mathrm{N}=21$ individuals) and two with X-linked recessive disorders ( $\mathrm{N}=2$ individuals). 35 loci were associated with disorders that can be inherited as either dominant or recessive conditions ( $N=88$ individuals).

\section{IEI Variants According to International Union of Immunologic Society Functional Class}

In this cohort we identified diverse IEI variants across all nine IUIS categories. Complement variants implicated in atypical hemolytic uremic syndrome (aHUS) were the most common, identified in 92 individuals (Fig. 3). The most common variants included $\mathrm{CFH}$ (c.3148A>T; p.Asn1050Tyr), C3 (c.1407G>C; p.Glu469Asp) and CFHR3 (c.424 C>T; p.Arg142Cys), all previously described in aHUS [2428]. We also identified other alternative complement variants reported in aHUS in CFHR5, CFI, CD46, CFB, CFHR3, THBD and CFHR4.

The next most frequent variant group was related to autoinflammatory conditions (Fig. 3). They occurred in 77 individuals and included variants reported in familial Mediterranean fever (MEFV), familial cold inflammatory syndrome type 2 (NLRP12), TNF receptor-associated periodic syndrome (TNFRSF1A), Blau syndrome (NOD2), pyogenic sterile arthritis, pyoderma gangrenosum acne syndrome (PSTPIP1) and CARD14-mediated pustular psoriasis (CARD14). The most common variant in this group, NLRP3 c. $2113 \mathrm{C}>A$; p.GIn705Lys was carried by 21 individuals and homozygous in one.

The remaining other pathogenic or potentially pathogenic variants according to IUIS Classification of Primary Immunodeficiencies include: combined immunodeficiencies with associated or syndromic features (KMT2D, CHD7, ERBB2IP, TTC37, SPINK5, TBX1, KMT2A); congenital defects of phagocyte number and function (ELANE, CFTR, ITGB2, GATA2); disorders of immune dysregulation (UNC13D, AIRE, $P R F 1, F A S, C A S P 10, X I A P, C T L A 4)$; defects of innate and intrinsic immunity (IRF3, STAT1, IL17RA, POLR3C, IRF4, TICAM1); bone marrow failure (TERT, RTEL 1, SRP72, SKIV2L, FANCG, FANCD, TINF2, ACD); predominantly antibody deficiencies (TNFRSF13B, TCF3, SLC39A); and mutations affecting cellular and humoral immunity (TAP1, REL, POLD1, IL2RG) as shown in Fig. 3. Variant data including zygosity, predicted in silico impact and MAF can be found in Supplemental Table S3. Phenotypic details for novel variants are found in Table S4.

IEI Status and IUIS Group Association with Infection Site and Laboratory Markers of Inflammation 
IEl-variants associated with infection site (Fig. 4a). Children with variants had increased odds of positive blood or urinary culture (blood: OR 2.82, 95\% Cl $1.12-7.10, p=0.022$; urine: OR: 8.23, 95\% Cl $1.06-$ $64.11, p=0.016$ ) and were less likely to have culture negative sepsis (OR: $0.59,95 \% \mathrm{Cl}: 0.38-0.92, p=$ 0.020). There was no significant difference in odds of positive respiratory culture or respiratory viral testing. As seen in Fig. 4b, Children with IEl-associated variants were more likely to be lymphopenic (OR: 1.67, 95\% Cl: $1.05-2.60, p=0.027$ ), hyperferritinemic (OR: 2.16, Cl: $1.28-3.67, p=0.004$ ), thrombocytopenic (OR: $1.76,95 \% \mathrm{Cl}: 1.12-2.76, p=0.013)$ and have a CRP > 10mg/dl (OR: 1.71, 95\% Cl: $1.09-2.68, p=0.017)$.

In comparison to children without identified variants, infection type and inflammatory markers also varied by IUIS Group (Fig. 4c). Individuals with variants from multiple IUIS classes had increased odds of positive blood, respiratory or urine culture and increased odds of hyperferrtinemia, lymphopenia, thrombocytopenia and CRP $>10 \mathrm{mg} / \mathrm{dL}$. Children with complement variants were less likely to have culture negative sepsis and had increased odds of hyperferritinemia, thrombocytopenia and CRP > $10 \mathrm{mg} / \mathrm{dL}$. Individuals with autoinflammatory variants had increased odds of positive urine culture. Individuals with combined immunodeficiencies with syndromic features had increased odds of positive blood or respiratory cultures, hyperferrtinemia, thrombocytopenia and CRP $>10 \mathrm{mg} / \mathrm{dl}$. Those innate immune disorder variants had increased odds of positive blood culture and hyperferritinemia, while those with phagocytic disorder variants had increased odds of positive urine culture. Individuals with immune dysregulation variants had the highest odds of hyperferritinemia seen in any group (OR 7.00: $95 \% \mathrm{Cl}: 2.3$ - 21.1, $p=0.0005$ ). Those with antibody deficiencies had increased odds of positive urine culture and those with variants affecting cellular and humoral immunity had decreased odds of culture negative sepsis. Following adjustment for multiple testing the following comparisons remained significantly different from children with no variants: 1.) Any variant: hyperferritinemia; 2.) Multiple variants: culture negative, blood culture positive, hyperferritinemia; 3 .) Complement: hyperferritinemia; 4.) Combined Syndromic: hyperferritinemia; 5.) Innate: hyperferritinemia; 6.) Phagoctye: positive urine culture; 7.) Dysregulation: hyperferritinemia (Tables S5 \& S6).

\section{Variants Overrepresented in the Pediatric Sepsis Cohort Compared to gnomAD}

After candidate variant identification, we sought statistical evidence of variant overrepresentation in children with severe sepsis compared to gnomAD [22] (Table 2, Table S7). Three complement variants were significantly over-represented: $C 3 \mathrm{c} .1407 \mathrm{G}>\mathrm{C}$; p.Glu469Asp (adjusted $p=1.2 \times 10^{-7}$ ), C3 c.443G>A; p.Arg148GIn (adjusted $p=4.5 \times 10^{-7}$ ) both activating variants of the main complement convertase [27] and CFHR3 c.424C>T; p.Arg142Cys (adjusted $p=2.6 \times 10^{-3}$ ), a negative regulator of complement [29]. In addition, IRF3 (c.829G>A; p.Ala277Thr), a transcriptional regulator of type I interferon (IFN)-dependent immune responses [30], was over-represented in the sepsis cohort (adjusted $p=0.012$ ).

\section{Variants Overrepresented in African American Children with Severe Sepsis}


After demonstrating that African American children had increased odds of variant identification, we sought to identify specific contributing variants. In African American children with severe sepsis, complement variants represented a higher proportion of variants than in the complete cohort (Fig. 5, $\mathrm{N}=38 / 70$ versus Fig. 3, $\mathrm{N}=92 / 330, p<0.0002$ ). When comparing African American to Non-African American children in our cohort, the activating convertase variant $C 3$ (c.1407G $>C$; p.Glu469Asp), complement regulatory variants CFHR3 (c.424C>T; p.Arg142Cys) and CFHR5 (c.434G>A; p.Gly145Glu), and TNFRSF1A (c.224C>T; p.Pro75Leu) an autoinflammation variant associated with increased NF-kB p65 activity and IL-8 secretion [31] were overrepresented (Table 2). Next, we compared MAF in African American children with sepsis to individuals of African descent in gnomAD, highlighting potential predisposing factors for severe sepsis among groups of similar ancestral background. While limited by small numbers, we saw that $C 3$ (c. 443G>A; p.Arg148GIn) and $C F H$ (c.2850G >T; p.GIn950His) were both more common than expected in African American children with sepsis (Table 2).

\section{Discussion}

In our severe sepsis cohort, over half of children had at least one IEl-linked variant. While overestimating immunodeficiency, we suggest this accurately estimates the proportion of children with genetic risk for immune dysfunction during infection as evidenced by increased odds of positive blood or urine culture, hyperferritinemia, thrombocytopenia, lymphopenia, elevated CRP and increased ECMO use. While septic episodes may rarely identify children with immunodeficiency, future study of the impact of these variants on host response to infection may provide mechanistic insight into the sepsis-related dysregulated immune response and inform mechanism-based immunomodulatory therapy. This may be especially true in tertiary intensive care units in resource rich settings, as their patients may represent a significantly enriched population because of immunization practices, early and aggressive antibiotic treatment, and low rates of endemic infection.

In a recent report, Borghesi et al. used WES to identify immunodeficiency variants in $20 \%$ of previously healthy children with positive blood cultures, predominantly described as variants of uncertain significance [8]. While these study participants had bacteremia, only $38 \%$ were admitted to ICU and less than half had organ dysfunction, a necessary criterion for diagnosis of severe sepsis. Their variant filtering included use of an inhouse database, in silico modeling, and private literature search contributing to differences in prevalence estimates. Still, both studies suggest that variation in immunodeficiency genes is associated with pediatric sepsis. Additionally, in our study of severe pediatric sepsis, we were further able to associate genetic findings with infection site, inflammatory response, and organ support with ECMO. Our pediatric findings are consistent with our previous report that six of six adults with extreme hyperferritinemic sepsis had pathogenic and potentially pathogenic IEI variants [32].

In all children with severe sepsis and in septic African American children specifically, complement variants were frequent. As part of innate immunity, complement functions in early pathogen response. Inactivating variants increase susceptibility to bacteria[4,33] and can be selected against in settings of endemic infection [34]. However, improved pathogen clearance may increase inflammation and 
thrombotic microangiopathy [35]. In this regard, C3 c.1407G $>C$ and CFHR3 c.424C $>T$, the most common variants in our cohort, have been reported as causal for aHUS $[27,29]$ and were statistically overrepresented in comparison to gnomAD. In addition to their reports in aHUS, other complement variants have also been observed in additional thrombotic phenotypes [36] including recurrent pregnancy loss (C3 c.2203C >T) [37], HELLP syndrome (CD46 c.1058C>T, CD46c.38C>T) [38,39] and drug-induced thrombotic microangiopathy (CFH c.2850G >T, CFH c.3148A $>$ T) [40]. Our observation that children with complement variants had increased odds of thrombocytopenia, hyperferritinemia and having a CRP >10 $\mathrm{mg} / \mathrm{dL}$ suggests that these variants convey risk for microangiopathy during episodes of severe sepsis.

When considering variant frequency, it is important to emphasize ancestry-specific differences. For C3 c. $1407 \mathrm{G}>\mathrm{C}, C F H R 3$ c.424C $>\mathrm{T}, C F H R 5$ c.434G $>\mathrm{A}$ and TNFRSF1A c. $224 \mathrm{C}>\mathrm{T}$, a TNFa receptor that directly activates complement signaling [41], overrepresentation may be partially explained by their frequency in African Americans. Still, complement overrepresentation is not completely explained by population stratification, as when African American children with serve sepsis are compared to African gnomAD participants, $C 3$ c.443G $>A$ and $C F H$ c.2850G $>$ T remained overrepresented. Acknowledging that African gnomAD participants are an imperfect control, previous reports of variants' disease impact emphasize complement's biologic relevance in sepsis pathobiology (Table S2). These findings lead us to hypothesize that observed differences in pediatric sepsis outcomes that associate with ancestry may be impacted by complement genetics and emphasize the need for enrollment of diverse cohorts in future genetic studies of severe pediatric sepsis.

The second most common functional group was autoinflammation variants. NLRP3 variants cause the cryopyrin-associated periodic syndromes including familial cold inflammatory syndrome, Muckle-Wells syndrome and neonatal-onset multisystem inflammatory disorder (NOMID). These disorders are monogenic inflammasomopathies inherited in an autosomal dominant pattern with incomplete penetrance. The specific NLRP3 p.GIn705Lys variant leads to constitutive hyperactivation with increased IL-1b and IL-18 synthesis [42] that associates with severity of acute phase response [43]. We also commonly observed MEFV variants, previously reported in familial Mediterranean fever that have been associated with an exaggerated inflammatory response to infection with larger increases in WBC count, ESR and LDH levels, more pronounced tachycardia and hypotension [44].

IRF3 (c.829G >A; p.Ala277Thr) variants were also encountered more commonly in the pediatric sepsis cohort than expected (adjusted $p=0.013$ ). Heterozygous IRF3 variants (c.829G $>A$; p.Ala277Thr) have been described in herpes simplex encephalitis and peripheral blood mononuclear cells isolated from individuals with IRF3 c. 829G >A have significantly lower CXCL10 and IFN-b levels following poly(I:C), HSV-1 (a DNA virus) and RNA virus exposure, suggesting impaired antiviral response [30]. Additionally, other IRF3 variants are overrepresented among individuals with life-threatening SARS-CoV-2 infection, where they are hypothesized to impair viral clearance [45]. As viral infections are a common cause of sepsis in children, this leads us to hypothesize that genetic interferon pathway variation may associate with risk for viral sepsis in general; however, as the absolute number of children with IRF3 variants in the cohort was small $(\mathrm{N}=9)$, we did not detect an increased rate of viral sepsis. 
Other key findings include that if offered in the PICU, genetic testing for immunologic disease is agreed to by $95 \%$ of parents of children with sepsis. The acceptability of genetic testing is important in light of questions surrounding patient and family preferences. We also found insufficient sampling of DNA was common in children with lymphopenia, suggesting a role for buccal mucosa, saliva or uroepithelial cell sampling techniques. This is of considerable importance, as sepsis patients with lymphopenia are at greater risk of morbidity and mortality [18]. Children with malignancy are also commonly lymphopenic, limiting the generalizability of our findings to this common sepsis subpopulation.

Our study's main limitation is that while the presence of a pathogenic or potentially pathogenic variant in a disease consistent inheritance pattern is remarkable, it cannot be equated with immunodeficiency. While candidate variants were restricted to those with prior associations with human disease, literaturebased classification is likely to misclassify a portion of variants and cannot be equated to clinical sequencing. We used the HGMD professional database for variant assignment, a resource curated to minimize false negatives, that consequently increases false positives in assignment of pathogenicity. Additionally, it is known that even well-established pathogenic variants do not always cause disease due to variable penetrance, expressivity, epistasis, gene-gene interactions, and environmental factors [10]. Therefore, even pathogenic (DM) variants for autosomal dominant conditions represent potential rather than confirmed immunodeficiencies. Additionally, the lack of long-term follow-up, functional immunologic testing, and characterization of dysmorphic features limits our ability to correlate our genetic findings to phenotype. However, the ability of our study to identify associations between IEI and type of infection, inflammatory response and need for extracorporeal therapy despite the noise introduced by incorrect assignment, emphasizes the function relevance of IEl in pediatric sepsis, while acknowledging that this analysis cannot be interpreted as evidence for individual variant pathogenicity. The enrollment of children with only severe sepsis and organ failure also limits the generalizability of our findings to septic children without organ failure. However, those with organ failure disproportionately represent sepsis-related morbidity and mortality, emphasizing a need for further study of the impact of IEI variants on host-pathogen interactions, the dysregulated host response, and need for organ support therapy once infection is established. Other notable limitations include the 10 cases of potential autosomal recessive biallelic disease, where WES is unable to differentiate cis from trans variants in the absence of parental sampling. WES also fails to identify regulatory, structural and copy number variants. Future study is needed to determine the impact of these variants on predisposition to infection, dysregulated host response, illness severity and recurrence risk between ancestry groups and among individuals with shared genetic risk.

\section{Conclusions}

In conclusion, genetic variation previously linked to inborn errors of immunity is common in our pediatric severe sepsis cohort. These variants were associated with bacteremia, urinary tract infection, laboratory markers of inflammation and requirement for extracorporeal membrane oxygenation. This suggests that evaluation of children with severe sepsis is warranted by clinical geneticists to screen for primary 
immunodeficiencies. Future study of heritable immunologic differences in children with severe sepsis may enable a genome-driven approach to the dysregulated host response.

\section{Declarations}

\section{Acknowledgements}

Clinical Research Investigation and Systems Modeling of Acute illness center: Ali Smith, BS; Octavia Palmer, MD; Vanessa Jackson, AA; Renee Anderko, BS, MS. Children's Hospital of Pittsburgh: Jennifer Jones, RN; Luther Springs. Children's Hospital of Philadelphia: Carolanne Twelves, RN, BSN, CCRC; Mary Ann Diliberto, BS, RN, CCRC; Martha Sisko, BSN, RN, CCRC, MS; Pamela Diehl, BSN, RN; Janice Prodell, RN, BSN, CCRC; Jenny Bush, RNC, BSN; Kathryn Graham, BA; Kerry Costlow, BS; Sara Sanchez. Children's National Medical Center: Elyse Tomanio, BSN, RN; Diane Hession, MSN, RN; Katherine Burke, BS. Children's Hospital of Michigan: Ann Pawluszka, RN, BSN; Melanie Lulic, BS. Nationwide Children's Hospital: Lisa Steele, RN, CCRC; Andrew R. Yates, MD; Josey Hensley, RN; Janet Cihla, RN; Jill Popelka, RN; Lisa Hanson-Huber, BS. Children's Hospital of Los Angeles and Mattel Children's Hospital: Jeni Kwok, JD; Amy Yamakawa, BS. Children's Hospital of Washington University of Saint Louis: Michelle Eaton, RN. Mott Children's Hospital: Frank Moler, MD; Chaandini Jayachandran, MS, CCRP. University of Utah Data Coordinating Center: Teresa Liu, MPH, CCRP; Jeri Burr, MS, RN-BC, CCRC, FACRP; Missy Ringwood, BS, CMC; Nael Abdelsamad, MD, CCRC; Whit Coleman, MSRA, BSN, RN, CCRC. This project used the University of Pittsburgh HSCRF Genomics Research Core's whole exome sequencing services (RRID: SCR_018301).

\section{Funding}

Supported, in part, by grant R01GM108618 (to Dr. Carcillo) from the National Institutes of General Medical Sciences, by grants K12HD047349, L30Al147146 (to Dr. Kernan) and 5U01HD049934-10S1 (to Dr. Carcillo) from the Eunice Kennedy Shriver National Institutes of Child Health and Human Development, National Institutes of Health, Department of Health and Human Services and the following cooperative agreements: U10HD049983, U10HD050096, U10HD049981, U10HD063108, U10HD63106, U10HD063114, U10HD050012, and U01HD049934.

\section{Conflicts of interest/Competing interests}

Drs. Carcillo's, Berg's, Wessel's, Pollack's, Meert's, Hall's, Doctor's, Cornell's, Harrison's, Zuppa's, Reeder's, Banks's, and Holubkov's institutions received funding from the National Institutes of Health (NIH). Drs. Carcillo's, Newth's, Shanley's, and Dean's institutions received funding from the National Institutes of Child Health and Human Development. Drs. Carcillo, Berg, Wessel, Polack, Meert, Hall, Newth, Doctor, Shanley, Cornell, Harrison, Zuppa, Reeder, Banks, Holubkov, Notterman, and Dean received support for article research from the NIH. Dr. Carcillo's institution also received funding from the National Institutes of General Medical Sciences. Dr. Pollack disclosed that his research is supported by philanthropy from Mallinckrodt Pharmaceuticals. Dr. Hall received funding from Bristol Myers-Squibb (for service on an advisory board) and LaJolla Pharmaceuticals (service as a consultant), both unrelated to the current 
submission. Dr. Newth received funding from Philips Research North America. Dr. Doctor's institution received funding from the Department of Defense and Kalocyte. Dr. Shanley received funding from Springer publishing, International Pediatric Research Foundation, and Pediatric Academic Societies. Dr. Cornell disclosed he is co-founder of Pre-Dixon Bio. Dr. Holubkov received funding from Pfizer (Data Safety Monitoring Board [DSMB] member), Medimmune (DSMB member), Physicians Committee for Responsible Medicine (biostatistical consulting), DURECT Corporation (biostatistical consulting), Armaron Bio (DSMB past member), and St Jude Medical (DSMB past member). Dr. Notterman and Dr. Kernan received funding from the National Institutes of Health. The remaining authors have disclosed that they do not have any potential conflicts of interest.

\section{Availability of data and material}

All data used in this analysis is available in the Main and Extended Tables.

\section{Code availability}

Not applicable.

\section{Authors' contributions}

This study was conceptualized by JAC, DAN, JMD, JL, UC and JV. Data curation was performed by KK, RS, DH, UC and RB. KK, LGG, RB, HJP, RWR performed formal analysis. Funding was acquired by JAC. The Investigation was performed by KK, JL, DH, UC, RS, JAC, RAB, DW, MMP, KM, MH, CN, JCL, AD, TS, TC, REH, AFZ, JMD. Methodology was created by JAC, JV, JL, JMD, DAN. Supervision of the project was performed by JAC, JV, LGG, JMD, DAN. Data visualization and original draft writing was performed by KK. All authors provided critical feedback and helped shape the research, analysis and manuscript.

\section{Ethics approval}

The study was approved by the central Institutional Review Board and all 9 individual site Institutional Review Boards

\section{Consent to participate}

Written informed consent was obtained from one or more parents/guardians for each child. Written assent was garnered when the child was able.

\section{Consent for publication}

Written informed consent was obtained was obtained for participation in the study, as well as consent for publication of study results.

\section{References}


1. Rudd KE, Johnson SC, Agesa KM, Shackelford KA, Tsoi D, Kievlan DR, et al. Global, regional, and national sepsis incidence and mortality, 1990-2017: analysis for the Global Burden of Disease Study. The Lancet. 2020;395:200-11.

2. Casanova J-L. Severe infectious diseases of childhood as monogenic inborn errors of immunity. Proceedings of the National Academy of Sciences. 2015;112:201521651.

3. Schulert GS, Canna SW. Convergent pathways of the hyperferritinemic syndromes. International Immunology. 2018;30:195-203.

4. Gaschignard J, Levy C, Chrabieh M, Boisson B, Bost-Bru C, Dauger S, et al. Invasive pneumococcal disease in children can reveal a primary immunodeficiency. Clinical Infectious Diseases. 2014;59:244-51.

5. Asgari S, McLaren PJ, Peake J, Wong M, Wong R, Bartha I, et al. Exome sequencing reveals primary immunodeficiencies in children with community-acquired Pseudomonas aeruginosa sepsis. Front. immunol. 2016; https://doi.org/10.3389/fimmu.2016.00357.

6. van der Made Cl, Simons A, Schuurs-Hoeijmakers J, van den Heuvel G, Mantere T, Kersten S, et al. Presence of Genetic Variants Among Young Men With Severe COVID-19. JAMA. 2020; 324(7):663673.

7. Meyts I, Bucciol G, Quinti I, Neven B, Fischer A, Seoane E, et al. Coronavirus disease 2019 in patients with inborn errors of immunity: An international study. Journal of Allergy and Clinical Immunology. 2021;147:520-31.

8. Borghesi A, Trück J, Asgari S, Sancho-Shimizu V, Agyeman PKA, Bellos E, et al. Whole-exome sequencing for the identification of rare variants in primary immunodeficiency genes in children with sepsis: A prospective, population-based cohort study. Clinical Infectious Diseases. 2020; 71:E61423.

9. Tangye SG, Al-Herz W, Bousfiha A, Chatila T, Cunningham-Rundles C, Etzioni A, et al. Human Inborn Errors of Immunity: 2019 Update on the Classification from the International Union of Immunological Societies Expert Committee. J Clin Immunol. 2020; 40: 24-64.

10. Taeubner J, Wieczorek D, Yasin L, Brozou T, Borkhardt A, Kuhlen M. Penetrance and Expressivity in Inherited Cancer Predisposing Syndromes. Trends in Cancer. 2018; 4:718-28.

11. Petrikin JE, Cakici JA, Clark MM, Willig LK, Sweeney NM, Farrow EG, et al. The NSIGHT1-randomized controlled trial: Rapid whole-genome sequencing for accelerated etiologic diagnosis in critically ill infants. Npj Genomic Med. 2018; https://doi.org/10.1038/s41525-018-0045-8

12. Saunders CJ, Miller NA, Soden SE, Dinwiddie DL, Noll A, Alnadi NA, et al. Rapid whole-genome sequencing for genetic disease diagnosis in neonatal intensive care units. Sci Transl Med. 2012; https://doi.org/10.1126/scitranslmed.3004041.

13. Mestek-Boukhibar L, Clement E, Jones WD, Drury S, Ocaka L, Gagunashvili A, et al. Rapid Paediatric Sequencing (RaPS): Comprehensive real-life workflow for rapid diagnosis of critically ill children. Med Genet. 2018; https://doi.org/10.1136/jmedgenet-2018-105396 
14. Sanford EF, Clark MM, Farnaes L, Williams MR, Perry JC, Ingulli EG, et al. Rapid Whole Genome Sequencing Has Clinical Utility in Children in the PICU. Pediatr Crit Care Med. 2019; https://doi.org/10.1097/PCC.0000000000002056

15. Doughty L, Clark RSB, Kaplan SS, Sasser H, Carcillo J. sFas and sFas Ligand and Pediatric SepsisInduced Multiple Organ Failure Syndrome. Pediatr Res. 2002; https://doi.org/10.1203/00006450200212000-00018

16. KJ P, W L, S C, WK M. The Frequency of Discordant Variant Classification in the Human Gene Mutation Database: A Comparison of the American College of Medical Genetics and Genomics Guidelines and ClinVar. Lab Med. 2021;52:250-9.

17. Richards S, Aziz N, Bale S, Bick D, Das S, Gastier-Foster J, et al. Standards and guidelines for the interpretation of sequence variants: a joint consensus recommendation of the American College of Medical Genetics and Genomics and the Association for Molecular Pathology. Genet Med. 2015; https://doi.org/10.1038/gim.2015.30

18. Felmet KA, Hall MW, Clark RSB, Jaffe R, Carcillo JA. Prolonged Lymphopenia, Lymphoid Depletion, and Hypoprolactinemia in Children with Nosocomial Sepsis and Multiple Organ Failure. The Journal of Immunology. 2005;174:3765-72.

19. Bennett TD, Hayward KN, Farris RW, Ringold S, Wallace CA, Brogan T V. Very high serum ferritin levels are associated with increased mortality and critical care in pediatric patients. Pediatr Crit Care Med. 2011;12:e233-6.

20. Sekhon SS, Roy V. Thrombocytopenia in adults: A practical approach to evaluation and management. Southern Medical Journal. 2006;99:491-8.

21. Lobo SMA, Lobo FRM, Peres Bota D, Lopes-Ferreira F, Soliman HM, Mélot C, et al. C-Reactive Protein Levels Correlate With Mortality and Organ Failure in Critically III Patients. Chest. 2003;123:2043-9.

22. Karczewski KJ, Francioli LC, Tiao G, Cummings BB, Alföldi J, Wang Q, et al. The mutational constraint spectrum quantified from variation in 141,456 humans. Nature. 2020;581:434-43.

23. Osborne AJ, Breno M, Borsa NG, Bu F, Frémeaux-Bacchi V, Gale DP, et al. Statistical Validation of Rare Complement Variants Provides Insights into the Molecular Basis of Atypical Hemolytic Uremic Syndrome and C3 Glomerulopathy. Journal of immunology. 2018;200:2464-78.

24. Geerlings MJ, Volokhina EB, de Jong EK, van de Kar N, Pauper M, Hoyng CB, et al. Genotypephenotype correlations of low-frequency variants in the complement system in renal disease and age-related macular degeneration. Clinical Genetics. 2018;94:330-8.

25. Besbas N, Gulhan B, Soylemezoglu O, Ozcakar ZB, Korkmaz E, Hayran M, et al. Turkish pediatric atypical hemolytic uremic syndrome registry: initial analysis of 146 patients. BMC nephrology. 2017;18:6.

26. Fidalgo T, Martinho P, Pinto CS, Oliveira AC, Salvado R, Borràs N, et al. Combined study of ADAMTS13 and complement genes in the diagnosis of thrombotic microangiopathies using nextgeneration sequencing. Research and Practice in Thrombosis and Haemostasis. 2017;1:69-80. 
27. Schramm EC, Roumenina LT, Rybkine T, Chauvet S, Vieira-Martins P, Hue C, et al. Mapping interactions between complement $\mathrm{C} 3$ and regulators using mutations in atypical hemolytic uremic syndrome. Blood. 2015; https://doi.org/10.1182/blood-2014-10-609073

28. Chapin J, Eyler S, Smith R, Tsai H-M, Laurence J. Complement factor $\mathrm{H}$ mutations are present in ADAMTS13-deficient, ticlopidine-associated thrombotic microangiopathies. Blood. 2013;121:40123.

29. Zhang T, Lu J, Liang S, Chen D, Zhang H, Zeng C, et al. Comprehensive analysis of complement genes in patients with atypical hemolytic uremic syndrome. American Journal of Nephrology. 2016;43:160-9.

30. Mørk N, Kofod-Olsen E, Sørensen KB, Bach E, Ørntoft TF, Østergaard L, et al. Mutations in the TLR3 signaling pathway and beyond in adult patients with herpes simplex encephalitis. Genes and Immunity. 2015;16:552-66.

31. Nedjai B, Hitman GA, Church LD, Minden K, Whiteford ML, McKee S, et al. Differential cytokine secretion results from p 65 and c-Rel NF-KB subunit signaling in peripheral blood mononuclear cells of TNF receptor-associated periodic syndrome patients. Cellular Immunology. 2011;268:55-9.

32. Kernan KF, Ghaloul-Gonzalez L, Shakoory B, Kellum JA, Angus DC, Carcillo JA. Adults with septic shock and extreme hyperferritinemia exhibit pathogenic immune variation. Genes Immun. 2019; https://doi.org/10.1038/s41435-018-0030-3

33. Davila S, Wright VJ, Khor CC, Sim KS, Binder A, Breunis WB, et al. Genome-wide association study identifies variants in the $\mathrm{CFH}$ region associated with host susceptibility to meningococcal disease. Nature Genetics. 2010;42:772-6.

34. Van Den Broek B, Van Der Flier M, De Groot R, De Jonge MI, Langereis JD. Common Genetic Variants in the Complement System and their Potential Link with Disease Susceptibility and Outcome of Invasive Bacterial Infection. Journal of Innate Immunity. 2020;12:131-41.

35. Bu F, Borsa N, Gianluigi A, Smith RJH. Familial atypical hemolytic uremic syndrome: a review of its genetic and clinical aspects. Clinical \& developmental immunology. 2012;2012:370426.

36. Tang L, Wang HF, Lu X, Jian XR, Jin B, Zheng H, et al. Common genetic risk factors for venous thrombosis in the chinese population. American Journal of Human Genetics. 2013;92:177-87.

37. Mohlin FC, Gros P, Mercier E, Gris JCR, Blom AM. Analysis of C3 gene variants in patients with idiopathic recurrent spontaneous pregnancy loss. Front. Immunol. 2018; https://doi.org/10.3389/fimmu.2018.01813

38. Fang CJ, Fremeaux-Bacchi V, Liszewski MK, Pianetti G, Noris M, Goodship THJ, et al. Membrane cofactor protein mutations in atypical hemolytic uremic syndrome (aHUS), fatal Stx-HUS, C3 glomerulonephritis, and the HELLP syndrome. Blood. 2008;111:624-32.

39. Crovetto F, Borsa N, Acaia B, Nishimura C, Frees K, Smith RJH, et al. The genetics of the alternative pathway of complement in the pathogenesis of HELLP syndrome. J Matern Fetal Neonatal Med; 2012;25:2322-5. 
40. Chapin J, Eyler S, Smith R, Tsai H-M, Laurence J. Complement factor H mutations are present in ADAMTS13-deficient, ticlopidine-associated thrombotic microangiopathies. Blood. 2013;121:40123.

41. Liu J, Tan Y, Zhang J, Zou L, Deng G, Xu X, et al. C5aR, TNFa and FGL2 contribute to coagulation and complement activation in virus-induced fulminant hepatitis. Journal of Hepatology. 2015;62:354-62.

42. Verma D, Särndahl E, Andersson H, Eriksson P, Fredrikson M, Jönsson J-I, et al. The Q705K polymorphism in NLRP3 is a gain-of-function alteration leading to excessive interleukin-1 $\beta$ and IL-18 production. PloS one. 2012;7:e34977.

43. Aksentijevich I, D. Putnam C, Remmers EF, Mueller JL, Le J, Kolodner RD, et al. The clinical continuum of cryopyrinopathies: Novel CIAS1 mutations in North American patients and a new cryopyrin model. Arthritis \& Rheumatism. 2007;56:1273-85.

44. Koc B, Oktenli C, Bulucu F, Karadurmus N, Sanisoglu SY, Gul D. The rate of pyrin mutations in critically ill patients with systemic inflammatory response syndrome and sepsis: a pilot study. The Journal of Rheumatology; 2007;34:2070-5.

45. Zhang Q, Liu Z, Moncada-Velez M, Chen J, Ogishi M, Bigio B, et al. Inborn errors of type I IFN immunity in patients with life-threatening COVID-19. Science. 2020; https://doi.org/10.1126/science.abd4570

\section{Tables}

Table 1. Characteristics for Participants, individuals with and without inborn errors of immunity on whole exome sequencing 


\begin{tabular}{|c|c|c|c|c|}
\hline & All $(N=330)$ & $\begin{array}{l}\text { IEI WES positive } \\
(\mathrm{N}=200)\end{array}$ & $\begin{array}{l}\text { IEI WES negative } \\
(N=130)\end{array}$ & p-value \\
\hline \multicolumn{5}{|l|}{ Patient Characteristics } \\
\hline Age (years) & $5.3(1.3-11.9)$ & $5.9(1.5-12.3)$ & $4.9(0.9-10.5)$ & NS \\
\hline Male & $179(54.2 \%)$ & $113(56.5 \%)$ & $66(50.7 \%)$ & NS \\
\hline \multicolumn{5}{|l|}{ Race } \\
\hline White & $224(67.9 \%)$ & $128(64.0 \%)$ & $96(73.8 \%)$ & NS \\
\hline Black & $70(21.2 \%)$ & $53(26.5 \%)$ & $17(13.1 \%)$ & $0.004^{\mathrm{a}}$ \\
\hline Other & $17(5.2 \%)$ & $11(5.5 \%)$ & $6(4.6 \%)$ & NS \\
\hline Not Reported & $22(6.7 \%)$ & $10(5.0 \%)$ & $12(9.2 \%)$ & NS \\
\hline \multicolumn{5}{|l|}{ Ethnicity } \\
\hline Hispanic or Latino & $51(15.5 \%)$ & $25(12.5 \%)$ & $26(20 \%)$ & NS \\
\hline Not Hispanic or Latino & $266(80.6 \%)$ & $166(83.0 \%)$ & $100(77.7 \%)$ & NS \\
\hline Unknown & $13(3.9 \%)$ & $9(4.5 \%)$ & $4(3.1 \%)$ & NS \\
\hline Previously Healthy & $158(47.9 \%)$ & $89(44.5 \%)$ & $69(53.0 \%)$ & NS \\
\hline Malignancy & $23(7.0 \%)$ & $17(8.5 \%)$ & $6(4.6 \%)$ & NS \\
\hline \multicolumn{5}{|l|}{ Patient Outcomes } \\
\hline ICU Mortality & $28(8.5 \%)$ & $18(9.0 \%)$ & $10(7.7 \%)$ & NS \\
\hline ICU LOS, days & $12(7-20.0)$ & $12.0(7.0-22.0)$ & $11.5(6.0-18.0)$ & NS \\
\hline MV & $302(91.5 \%)$ & $181(90.5 \%)$ & $121(93.1 \%)$ & NS \\
\hline Intubated & $161(48.8 \%)$ & $99(49.5 \%)$ & $62(47.6 \%)$ & NS \\
\hline CRRT & 34 (10.3\%) & 23 (11.5\%) & $11(8.5 \%)$ & NS \\
\hline ECMO & $21(6.4 \%)$ & $18(9.0 \%)$ & $3(2.3 \%)$ & $0.019^{b}$ \\
\hline PLEX & $18(5.5 \%)$ & $12(6.0 \%)$ & $6(4.6 \%)$ & NS \\
\hline
\end{tabular}

Categorical data are expressed as $N(\%)$. Continuous variables are presented as median and (IQR). Statistical comparison between groups was performed using Wilcoxon-rank sum for continuous variables and ${ }^{2}$ test for categorical variables. There were no significant differences between the complete cohort 
and IEI positive or negative groups. In comparing children with severe sepsis with IEI variants to those without, children of African American ancestry were noted to have increased odds of having an IEl variant identified. Children with IEl variants also had increased odds of requiring extracorporeal membrane oxygenation (ECMO).
a. OR $2.3995 \% \mathrm{Cl} 1.31-4.36, p=0.004$
b. OR $4.19 \mathrm{Cl} 1.21-14.5, p=0.019$

IEI: Inborn Errors of Immunity; IQR: Interquartile range; N: number; ICU: intensive care unit; LOS: length of stay; MV: mechanical ventilation; ECMO: extracorporeal membrane oxygenation; PLEX: plasma exchange; CRRT: continuous renal replacement therapy

Table 2 Summary table of overrepresented inborn errors of immunity alleles by ancestry group following multiple test correction 


\begin{tabular}{|c|c|c|c|c|c|c|}
\hline Variant & $\begin{array}{l}\text { MAF Ratio: } \\
\text { Sepsis / } \\
\text { gnomAD, } \\
\text { (N) }\end{array}$ & $\begin{array}{l}\text { Adj } \\
p \text {-value }\end{array}$ & $\begin{array}{l}\text { MAF Ratio: } \\
\text { African } \\
\text { American } \\
\text { Sepsis / } \\
\text { Non- } \\
\text { African } \\
\text { American } \\
\text { Sepsis, (N) }\end{array}$ & $\begin{array}{l}\text { Adj } \\
p \text {-value }\end{array}$ & $\begin{array}{l}\text { MAF } \\
\text { Ratio: } \\
\text { African } \\
\text { American } \\
\text { Sepsis / } \\
\text { gnomAD } \\
\text { African, } \\
\text { (N) }\end{array}$ & $\begin{array}{l}\text { Adj } \\
p \text {-value }\end{array}$ \\
\hline $\begin{array}{l}\text { C3 c.1407G>C } \\
\text { p.Glu469Asp }\end{array}$ & $\begin{array}{l}4.7 \\
(N=14)\end{array}$ & $\begin{array}{l}1.2 \times 10^{-} \\
7\end{array}$ & $\begin{array}{l}22.3 \\
(N=12)\end{array}$ & $\begin{array}{l}4.4 \times 10^{-} \\
5\end{array}$ & & \\
\hline $\begin{array}{l}\text { C3 c. } 443 G>A \\
\text { p.Arg148GIn }\end{array}$ & $\begin{array}{l}214.2 \\
(N=1)\end{array}$ & $\begin{array}{l}4.5 \times 10^{-} \\
7\end{array}$ & & & $\begin{array}{l}0.014 / 0, \\
(N=1)\end{array}$ & $\begin{array}{l}4.08 \times 10^{-} \\
9\end{array}$ \\
\hline $\begin{array}{l}\text { CFH c. } 2850 G>T \\
\text { p.Gln950His }\end{array}$ & & & & & $\begin{array}{l}18.1,(\mathrm{~N}= \\
2)\end{array}$ & $\begin{array}{l}9.73 \times 10^{-} \\
6\end{array}$ \\
\hline $\begin{array}{l}\text { CFHR3 c.424C>T } \\
\text { p.Arg142Cys }\end{array}$ & $\begin{array}{l}2.9 \\
(N=15)\end{array}$ & $2.6 \times 10^{-3}$ & $\begin{array}{l}14.9 \\
(N=12)\end{array}$ & $2.3 \times 10^{-6}$ & & \\
\hline $\begin{array}{l}\text { CFHR5 c. } 434 \mathrm{G}>\mathrm{A} \\
\text { p.Gly } 145 \mathrm{Glu}\end{array}$ & & & $\begin{array}{l}29.7 \\
(N=8)\end{array}$ & $7.3 \times 10^{-5}$ & & \\
\hline $\begin{array}{l}\text { IRF3 c.829G }>\text { A } \\
\text { p.Ala277Thr }\end{array}$ & $\begin{array}{l}3.4 \\
(N=9)\end{array}$ & 0.012 & & & & \\
\hline $\begin{array}{l}\text { TNFRSF1A } \\
\text { c.224C>T } \\
\text { p.Pro75Leu }\end{array}$ & & & $\begin{array}{l}18.6 \\
(N=10)\end{array}$ & $1.4 \times 10^{-5}$ & & \\
\hline
\end{tabular}

Summary table of statistically significant frequency comparisons following multiple test corrections between groups: 1.) Sepsis cohort and gnomAD to identify variants more common in the sepsis population as a whole; 2.) African American and Non-African American children in the sepsis cohort to identify variants that may explain the increased odds of potential IEl variants identified in African American children; 3.) African Americans in the sepsis cohort and individuals of African ancestry in gnomAD to identify overrepresented variants on a specific ancestral background. Table shows ratio of minor allele frequency in statistically significant comparisons of interest and number of individuals within the subgroup with the variant. $P$ values shown are $c^{2}$ comparisons with Benjamini-Hochberg adjustment for the total number of unique variants observed in the dataset $(\mathrm{N}=131)$. 


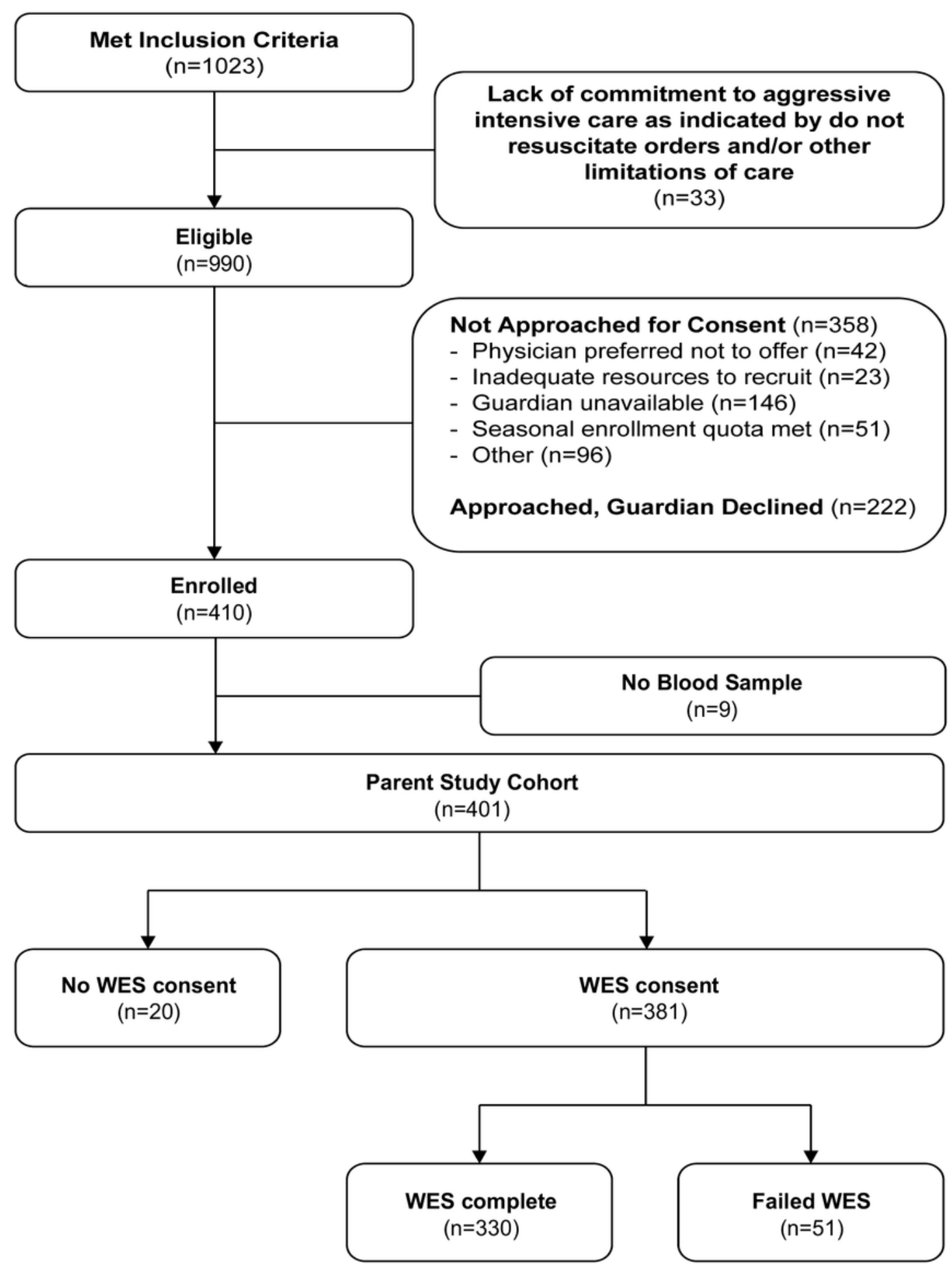

Figure 1

Consort diagram for study Diagram of recruitment, inclusion, and exclusion criteria. 


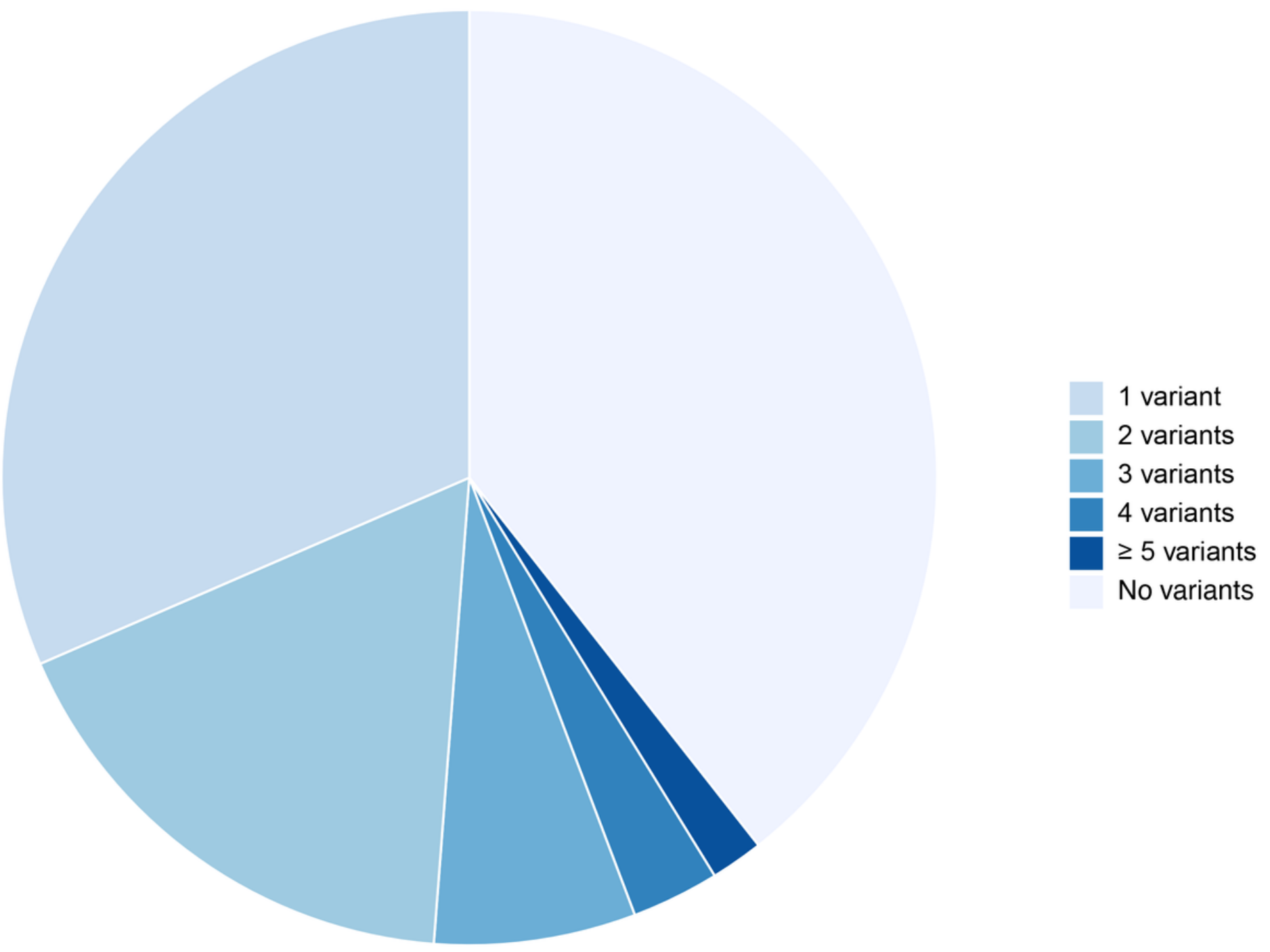

Figure 2

Distribution of number of variants per subject in the severe pediatric sepsis cohort Shows the number of inborn errors of immunity variants identified per subject, which ranged from zero to eight. 


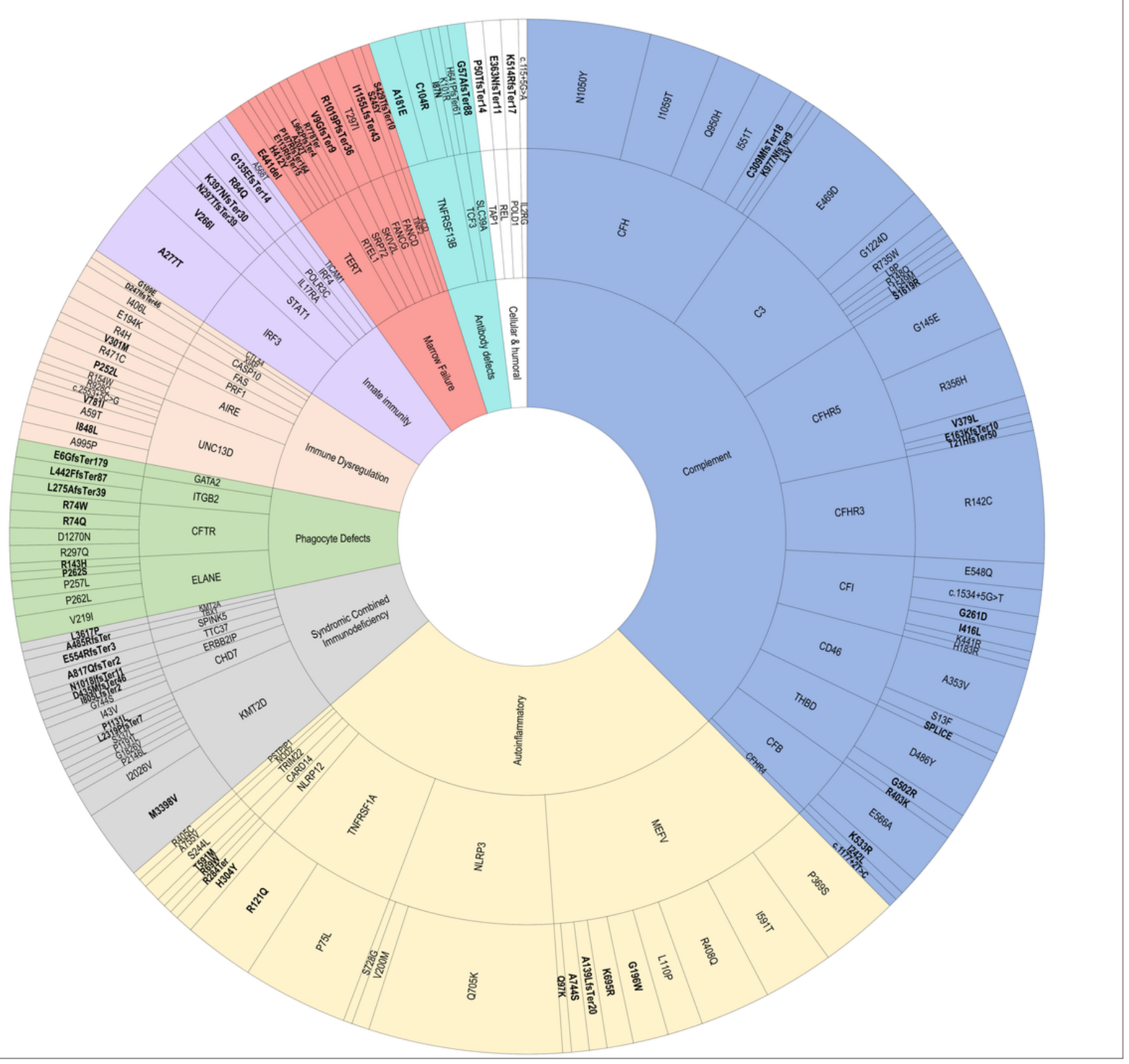

\section{Figure 3}

Genomic landscape of inborn errors of immunity in severe pediatric sepsis. This figure displays every pathogenic or potentially pathogenic variant identified in the cohort and from inner- to outer-most ring indicates its International Union of Immunologic Societies Classification, the disease where the variant has been previously identified, the gene locus affected and finally the specific allele change. 131 unique variants were identified in 200 individuals. Representatives of all IUIS disease classes were identified in the cohort and include from most to least common: complement deficiencies (complement, blue), autoinflammatory disorders (autoinflammatory, yellow), combined immunodeficiency with associated or syndromic features (syndromic features, gray), congenital defects of phagocyte number and function (phagocyte defects, green), diseases of immune dysregulation (immune dysregulation, peach), defects of 
innate and intrinsic immunity (innate immunity, purple), bone marrow failure disorders (marrow failure, red), predominantly antibody deficiencies (antibody, teal) and immunodeficiencies affecting cellular and humoral immunity (T \& B cell, white). Variants assigned DM in the HGMD database are shown in bold, while those with DM? designations are shown in plain font face. The wedge size allotted to an individual variant, gene, disease, and immune disorder classification is proportional to the relative frequency of its observation in the dataset.

a.

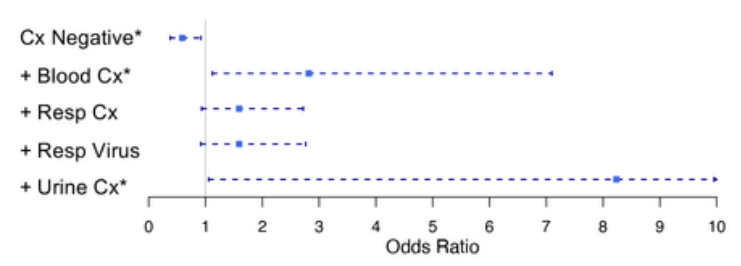

b.

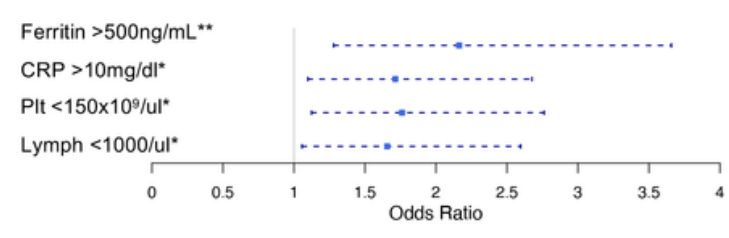

c.

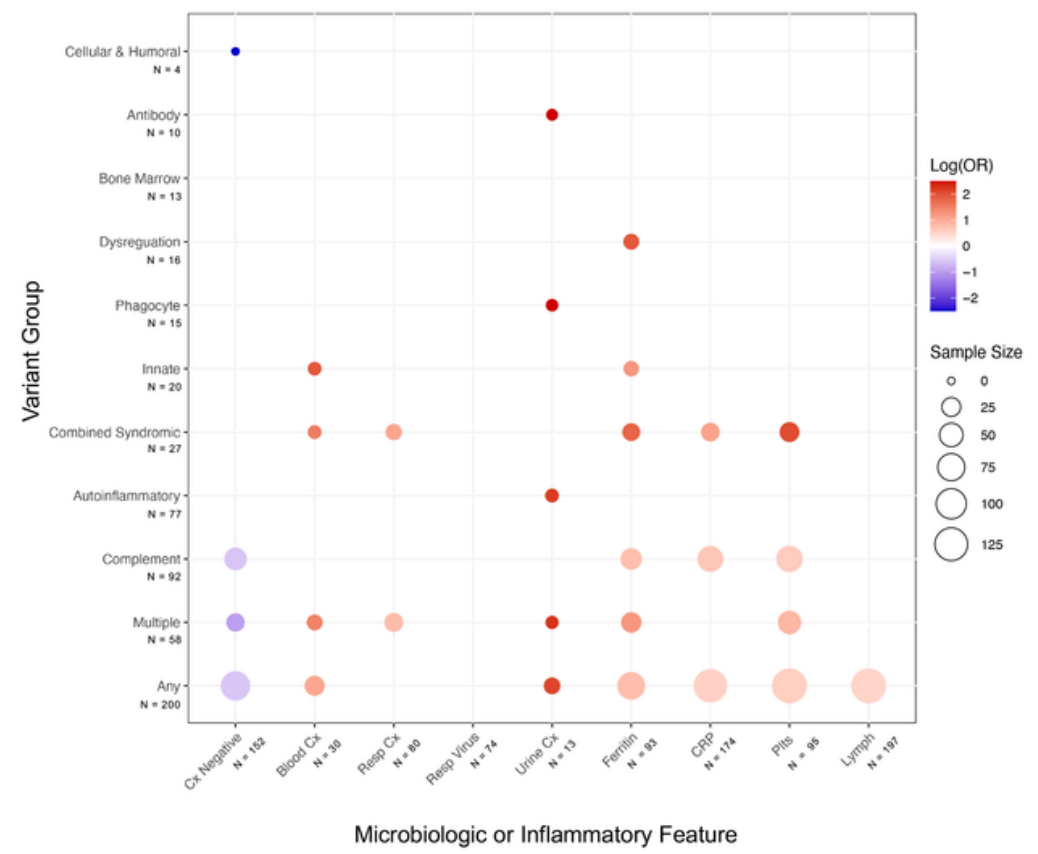

\section{Figure 4}

Infection types and immunologic characteristics for individuals with and without inborn errors of immunity a. Shows the odds ratios (OR) and $95 \% \mathrm{Cl}$ for microbiologic testing in children with any inborn error of immunity compared to children with no variants identified. Microbiologic data includes all viral testing and bacterial culture data +/- 2 days from enrollment with likely contaminants removed.

Respiratory cultures include specimens taken from tracheal aspirate, pleural fluid culture, bronchial brush and bronchoalveolar lavage. Children with variants were noted to have significantly increased odds of positive blood and urine culture, as well as decreased odds of culture negative sepsis. $b$. Shows the odds ratios and $95 \% \mathrm{Cl}$ for inflammatory laboratory characteristics in children with any inborn error of immunity compared to children with no variants identified. Comparison is of the most extreme values from any day on study. Children with potential inborn error of immunity variants were noted to have significantly increased odds of hyperferritinemia, elevated CRP, thrombocytopenia and lymphopenia. c. Shows the $\log (\mathrm{OR})$ for each microbiologic and inflammatory laboratory feature by IUIS variant group compared to children with no variant identified. Only ORs that differed significantly from children with no identified variants are shown ( $<<0.05$, unadjusted). Following adjustment for multiple test correction the following ORs remained significantly different from children with no variants identified: 1.) any: 
hyperferritinemia; 2.) multiple: culture negative, positive blood culture, hyperferritinemia; 3.) complement: hyperferritinemia; 4.) combined syndromic: hyperferritinemia; 5.) innate: hyperferritinemia; 6.) phagoctye: positive urine culture; 7.) dysregulation: hyperferritinemia). For example, children with any variant, variants from multiple IUIS classes, combined syndromic and innate immune IUIS classes had increased odds of positive blood cultures compared to children with no variants identified. Similarly, children with any variant, variants from multiple classes, complement variants and combined cellular \& humoral defects had decreased odds of having culture negative sepsis. Multiple: children with variants from more than one IUIS class, $\mathrm{Cx}$ : culture, ${ }^{\star} \mathrm{p}<0.05,{ }^{\star \star} \mathrm{p}<0.01$

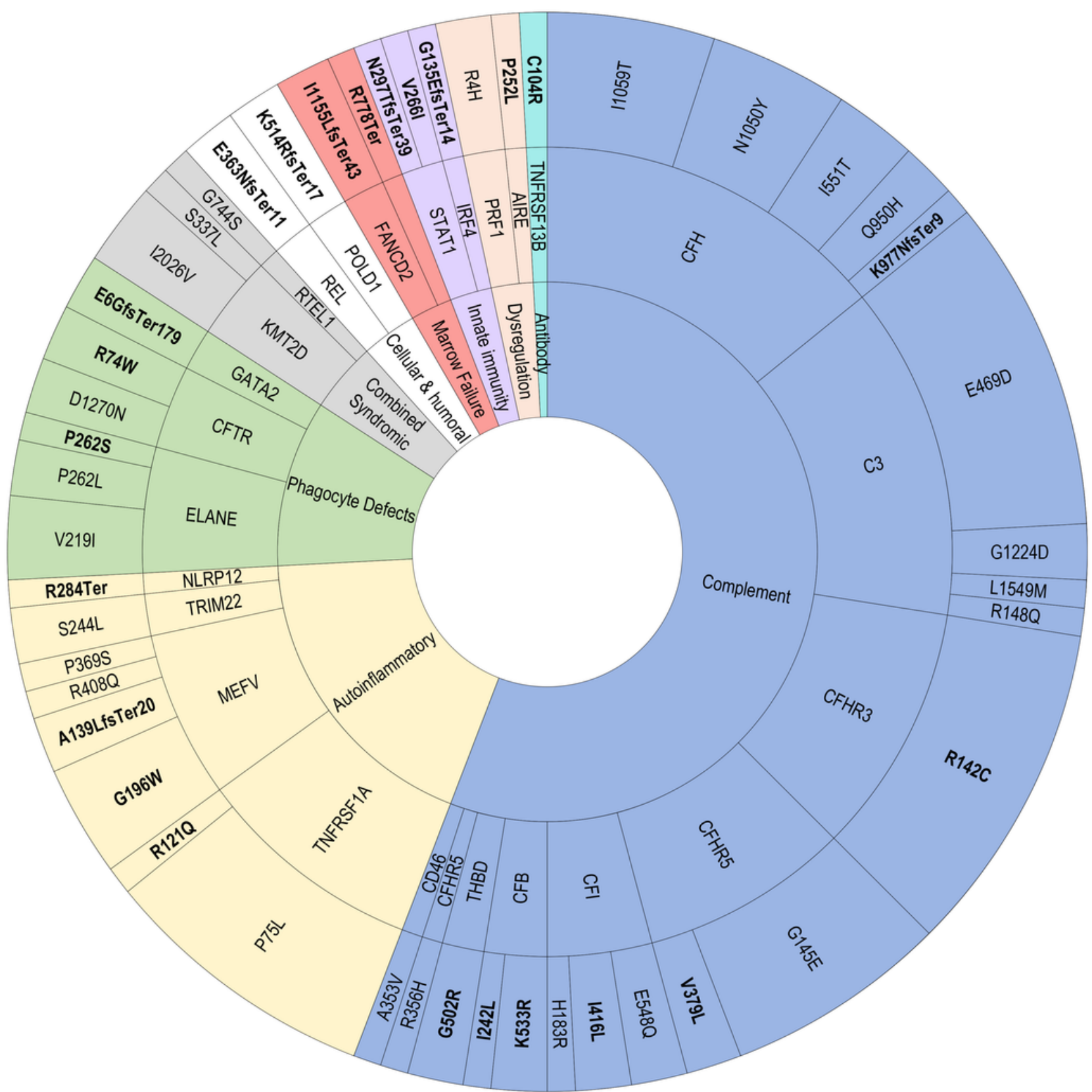

Figure 5 
Genomic landscape of inborn errors of immunity in African American children with severe pediatric sepsis This figure displays every pathogenic or potentially pathogenic variant identified in African American children with severe sepsis. From inner- to outer-most ring it indicates the variants' International Union of Immunologic Societies Classification, the human disease where it has been previously identified, the gene locus affected and finally the specific allele change. Representatives of all IUIS disease classes were identified in the cohort including from most to least common: complement deficiencies (complement, blue), autoinflammatory disorders (autoinflammatory, yellow), defects of phagocyte number and function (phagocyte defects, green), combined immunodeficiency with associated or syndromic features (syndromic features, gray), immunodeficiencies affecting cellular and humoral immunity (T \& B cell, white), bone marrow failure disorders (marrow failure, red), defects of innate and intrinsic immunity (innate immunity, purple), congenital diseases of immune dysregulation (immune dysregulation, peach), and predominantly antibody deficiencies (antibody, teal). Variants assigned DM in the HGMD database are shown in bold, while those with DM? designations are shown in plain font face. The wedge size allotted to an individual variant, gene, disease, and immune disorder classification is proportional to the relative frequency of its observation in the dataset.

\section{Supplementary Files}

This is a list of supplementary files associated with this preprint. Click to download.

- SupplementalS1Table2019IUISGeneList.xlsx

- SupplementalS2TableSupplementalreferencesbyvariant.docx

- SupplementalS3TableVariantsbysubject.xlsx

- SupplementalS4Tablephenotypedetailnovel.xls

- SupplementalS5TableORmicro.supplemental.table.pdf

- SupplementalS6TableOR.labs.supplemental.table.pdf

- SupplementalS7Table.FrequencyCohortandgnomAD.xlsx 